\title{
Respiratory Volume Monitoring Reduces Hypoventilation and Apnea in Subjects Undergoing Procedural Sedation
}

\author{
Robert H Nichols DO, Justin A Blinn MD, Thuan M Ho MD, Robert A McQuitty, and \\ Michael P Kinsky MD
}

\begin{abstract}
INTRODUCTION: The use of monitored anesthesia care for endoscopic procedures increases the risk of respiratory depression, necessitating careful monitoring of patient ventilation. We examined the effectiveness of an impedance-based respiratory volume monitor (RVM) in improving the safety of patients undergoing upper and lower gastrointestinal endoscopies under total intravenous anesthesia. We hypothesized that feedback from the RVM would allow anesthesiologists to maintain adequate ventilation, which would reduce the duration of respiratory depression (ie, hypoventilation and apnea) compared to a blinded control group. METHODS: Sixty-five subjects were enrolled in a randomized controlled trial and monitored with a noninvasive impedance-based RVM, which displayed respiratory traces and calculated expiratory minute ventilation $\left(\dot{\mathbf{V}}_{E}\right)$, tidal volume $\left(V_{T}\right)$, and breathing frequency (f) measurements. Prior to induction of anesthesia, a baseline $\dot{V}_{E}$ measurement $\left(\dot{\mathrm{V}}_{\text {E-baseline }}\right)$ was taken as a measurement of normal breathing. $\dot{\mathrm{V}}_{\mathbf{E}}$ was monitored throughout the procedure for signs of hypoventilation and apnea. Hypoventilation was defined as $\dot{\mathrm{V}}_{\mathrm{E}}<40 \% \dot{\mathrm{V}}_{\mathrm{E}-b a s e l i n e}$, and apneas were defined as $\dot{\mathrm{V}}_{\mathrm{E}}=0$ for $>15 \mathrm{~s}$. RESULTS: Sixty-five subjects were randomly assigned to either a control $(n=38)$ or RVM intervention group $(n=27)$. Subjects in the intervention group had a higher $\dot{V}_{E} \%$ for the entire procedure $(P=.045)$, as well as the third and fourth quartile of the procedure compared to the control group $(P=.01)$. Likewise, subjects in the RVM intervention group spent significantly less time below $40 \% \dot{\mathrm{V}}_{\mathrm{E} \text {-baseline }}$ compared to the control group throughout the entire procedure $(12 \pm 15 \%$ vs $32 \pm 24 \%$, respectively $)(P<.001)$. The median number of apneas per subject was greater in the control group (median 2, interquartile range 1-2, maximum 4) compared to the RVM intervention group (median 1, interquartile range 1-2, maximum 3) $(P=.037)$. CONCLUSIONS: The control group had a higher incidence of hypoventilation and apnea compared to the RVM intervention group. Respiratory monitoring using the RVM can potentially be a useful tool for identifying early signs of respiratory depression and for titrating anesthetics to maintain adequate ventilation while minimizing patient risk. Key words: endoscopy; procedural sedation; respiratory volume monitor; ventilation monitoring. [Respir Care 2018;63(4):448-454. (C) 2018 Daedalus Enterprises]
\end{abstract}

\section{Introduction}

Over 20 million endoscopic procedures are performed per year in the United States, with one third of them involving monitored anesthesia care. ${ }^{1,2}$ The increase in the use of monitored anesthesia care can be attributed partly to

Drs Nichols, Blinn, Ho, McQuitty, and Kinsky are affiliated with The University of Texas Medical Branch at Galveston, Galveston, Texas. Dr Ho is also affiliated with the Stanford University School of Medicine, Stanford, California. the growing use of propofol, which can provide a deeper level of sedation while also providing rapid onset and recovery times. ${ }^{2,3}$ Side effects of propofol include respiratory depression and apnea, which necessitate careful monitoring of ventilation to maintain patient safety. Respira-

\footnotetext{
The authors disclose no conflicts of interest.

Dr Ho presented a version of this paper at the International Anesthesia Research Society Annual Meeting, held May 21-24, 2016, in San Francisco, California.
} 
tory monitoring of non-intubated patients poses a challenge for anesthesiologists who must rely on secondary indicators of adequate ventilation. American Society of Anesthesiologists (ASA) guidelines strongly encourage the use of continuous monitoring equipment to augment visual observation of ventilation (ASA Standards for Basic Anesthetic Monitoring, www.asahq.com. Accessed August 23, 2017). Because the number of endoscopies during which propofol is used is expected to increase, ${ }^{1}$ continuous ventilation monitoring will be crucial for patient safety.

Analysis of the ASA closed-claims database revealed that $>40 \%$ of claims with monitored anesthesia care resulted in death or permanent brain damage, which is comparable to general anesthesia claims. ${ }^{4}$ The most prevalent cause of adverse events associated with monitored anesthesia care was oversedation leading to respiratory depression. Specifically, $84 \%$ of claims associated with oversedation resulted in death or permanent brain damage. Notably, $44 \%$ of claims involving oversedation were deemed preventable with better monitoring. ${ }^{4}$ Likewise, Metzner et $\mathrm{al}^{5}$ found that more than half of oversedation claims occurred in patients undergoing gastrointestinal endoscopies, of which a surprising $62 \%$ were judged to be preventable with better monitoring.

To minimize the incidence of adverse events, the ASA recommends the use of 2 continuous monitoring technologies: pulse oximetry and capnography. Pulse oximetry measures peripheral capillary oxygen saturation and can alert care providers to incidents of oxygen desaturation; however, the onset of respiratory depression precedes desaturation. ${ }^{6,7}$ The inherent delay in pulse oximetry alarms makes it a good indicator but a poor predictor of respiratory depression. Furthermore, the administration of supplemental oxygen can exacerbate the delay by masking cases of hypoventilation. ${ }^{8}$ While capnography is a reliable technique for measuring partial pressure of end-tidal $\mathrm{CO}_{2}$ pressure $\left(\mathrm{P}_{\mathrm{ETCO}_{2}}\right)$ in intubated patients, its use in procedural sedation has produced mixed results. ${ }^{9-12}$ A study by Sivilotti et al ${ }^{10}$ found that changes in $\mathrm{P}_{\mathrm{ETCO}_{2}}$ exhibited the same delay problem as measurements of oxygen saturation. Likewise, recent randomized controlled trials by van Loon et $\mathrm{al}^{11}$ and Campbell et al ${ }^{12}$ found no effect of capnography monitoring on incidence of hypoxemia during procedures with propofol sedation. Despite the use of pulse oximetry and capnography in procedural sedation, the risk of adverse events due to respiratory depression persists,

Correspondence: Michael P Kinsky MD, Department of Anesthesiology, University of Texas Medical Branch, Galveston, 301 University Blvd, Galveston, TX 77555. E-mail: mkinsky@utmb.edu.

DOI: $10.4187 /$ respcare. 05723

\section{QUICK LOOK}

\section{Current knowledge}

Monitoring respiratory status is critical for patients undergoing procedural sedation as they are at risk of respiratory depression resulting from oversedation. Current technologies such as pulse oximetry and capnography do not directly measure ventilation and are therefore delayed indicators of respiratory depression. Closed-claims analysis suggests that half of the claims resulting in death or permanent brain damage were preventable with better monitoring. Patient safety could greatly benefit from direct measurements of ventilation during procedural sedation.

\section{What this paper contributes to our knowledge}

We used a novel noninvasive respiratory volume monitor (RVM) to directly measure minute ventilation to direct care for subjects undergoing endoscopies under procedural sedation. Subjects of anesthesiologists who used the respiratory volume monitor to direct care maintained higher minute ventilation and had a lower incidence of hypoventilation and apnea compared to a control group in which anesthesiologists were blinded to respiratory volume monitor metrics.

and patient safety stands to improve from a more direct and responsive measure of ventilation.

A new noninvasive respiratory volume monitor (RVM) has been shown to overcome limitations associated with pulse oximetry and capnography. ${ }^{13-15}$ The impedance-based RVM provides continuous, real-time measurements of expiratory minute ventilation $\left(\dot{\mathrm{V}}_{\mathrm{E}}\right)$, tidal volume $\left(\mathrm{V}_{\mathrm{T}}\right)$ and breathing frequency (f), which are more direct measurements of respiratory activity compared to $\mathrm{S}_{\mathrm{pO}_{2}}$ and $\mathrm{P}_{\mathrm{ETCO}} \cdot{ }^{14-16}$ Specifically, the RVM can provide advance warning of respiratory depression ahead of alerts of oxygen desaturation by pulse oximetry, as well as identify oximeter false alarms due to patient motion. ${ }^{14}$ Compared to capnography, changes in $\dot{\mathrm{V}}_{\mathrm{E}}$ measured by the RVM are larger in degree and occur earlier than changes in $\mathrm{P}_{\mathrm{ETCO}_{2}}$ in response to respiratory fluctuations. ${ }^{15}$ Although the ability of the RVM to accurately measure $\dot{V}_{\mathrm{E}}, \mathrm{V}_{\mathrm{T}}$, and f and to detect hypoventilation has been verified, ${ }^{13}$ its use by anesthesiologists to improve patient safety has yet to be tested in a randomized controlled trial.

This study examined the effectiveness of using the RVM to improve the safety of patients undergoing upper and lower gastrointestinal endoscopies under total intravenous anesthesia with propofol and remifentanil. In particular, we measured changes in $\dot{\mathrm{V}}_{\mathrm{E}} \%$ to determine whether the incidence rate of hypoventilation and apnea decreased when 
anesthesiologists were encouraged to titrate total intravenous anesthesia according to the RVM. We hypothesized that feedback from the RVM would allow anesthesiologists to maintain adequate ventilation, which would reduce the rate of hypoventilation and apnea compared to a blinded control group.

\section{Methods}

This study was conducted as a randomized clinical trial during a clinical evaluation of the RVM as a device for monitoring total intravenous anesthesia cases at University of Texas Medical Branch. Participants undergoing upper and lower gastrointestinal endoscopies were included in July and December of 2015. Inclusion criteria were men and women aged 18 to $99 \mathrm{y}$, while exclusion criteria were non-English speakers, allergies to adhesive electrodes, and previous pneumonectomy or lobectomy surgery. Because this study was part of a clinical evaluation in which the use of the RVM was part of standard of care, subject data were collected retrospectively. The University of Texas Medical Branch Institutional Review Board approved the study and waived the requirement to obtain informed consent in accordance with 45 CFR 46.116(d). This study was supported in part by U.S. Army grant W81XWH-12-1-0598.

\section{Respiratory Volume Monitor}

The respiratory status of subjects was monitored using a noninvasive impedance-based RVM (ExSpiron 1Xi, Respiratory Motion, Waltham, Massachusetts), which can display respiratory traces and calculate $\dot{V}_{\mathrm{E}}, \mathrm{V}_{\mathrm{T}}$, and f measurements in real time. The impedance signal was recorded via the electrode PadSet, which was placed on the subject's thoracic region at the sternal notch, xiphoid, and mid-axillary line at the level of the xiphoid, according to manufacturer's specifications. ${ }^{13}$ After calibration, the RVM has demonstrated high accuracy compared to spirometry, with an average error of $<10 \%$ for $\dot{\mathrm{V}}_{\mathrm{E}}$ and $\mathrm{V}_{\mathrm{T}}$ and $<2 \%$ for $\mathrm{f}^{13}$ In this study the RVM was used in percent baseline mode, which does not include a calibration step with a spirometer. Instead a baseline impedance signal was recorded for $30 \mathrm{~s}$ during quiet breathing with the subject in position for the endoscopy procedure but before induction of anesthesia. The baseline measurement of $\dot{V}_{\mathrm{E}}\left(\dot{\mathrm{V}}_{\mathrm{E} \text {-baseline }}\right)$ was calculated, and all subsequent measurements were displayed as a percentage of $\dot{\mathrm{V}}_{\mathrm{E}-\text { baseline }}\left(\dot{\mathrm{V}}_{\mathrm{E}} \%\right)$ according to the formula $\dot{\mathrm{V}}_{\mathrm{E}} \%=\dot{\mathrm{V}}_{\mathrm{E} \text {-measured }} / \dot{\mathrm{V}}_{\mathrm{E} \text {-baseline }} \times 100 \% . \dot{\mathrm{V}}_{\mathrm{E}} \%$, $\mathrm{V}_{\mathrm{T}} \%$, and $\mathrm{f}$ were calculated from 30-s overlapping windows and updated every $5 \mathrm{~s}$. The anesthesiologist was presented with the plots of the respiratory impedance trace, $\dot{\mathrm{V}}_{\mathrm{E}} \%, \mathrm{~V}_{\mathrm{T}} \%$, and $\mathrm{f}$ along with a readout of current values for $\dot{\mathrm{V}}_{\mathrm{E}} \%, \mathrm{~V}_{\mathrm{T}} \%$, and $\mathrm{f}$. To make the $\dot{\mathrm{V}}_{\mathrm{E}} \%$ signal more readable, the plot of $\dot{V}_{\mathrm{E}} \%$ also indicated the range of low
$\dot{\mathrm{V}}_{\mathrm{E}} \%(<40 \%)$ and adequate $\dot{\mathrm{V}}_{\mathrm{E}} \%(40-80 \%)$ with orange and yellow backgrounds, respectively. A low $\dot{\mathrm{V}}_{\mathrm{E}} \%$ alarm was set by default at $40 \%$ and sounded immediately when the $\dot{V}_{\mathrm{E}} \%$ dropped below this threshold. Anesthesiologists were trained on the RVM prior to using it in the study.

\section{Randomized Control Trial and Total Intravenous Anesthesia Dosing Protocols}

Sixty-five subjects were enrolled into this randomized controlled trial, where they were randomly assigned based on the day of the week of the procedure to either the control group (Monday and Wednesday) or the intervention group (Tuesday and Thursday). Subjects in the control group were monitored with the RVM; however, the anesthesiologist remained blinded to the respiratory measurements throughout the procedure. Conversely, anesthesiologists in the intervention group were encouraged to use the RVM to titrate total intravenous anesthesia to maintain $\dot{\mathrm{V}}_{\mathrm{E}} \%$ within the range of $40-80 \%$, where subjects demonstrated some decrease in ventilation associated with sedation but did not demonstrate respiratory depression. Anesthesiologists were evenly distributed among the control and intervention groups.

Total intravenous anesthesia with propofol was performed in all subjects along with remifentanil in a subset of subjects using standard protocols in the control group, with a minor adjustment in the intervention group. Standard ASA monitoring included noninvasive blood pressure, heart rate by electrocardiogram, and arterial pulse oxygen saturation and capnography were used to determine the breathing frequency and signs of airway obstruction. $\mathrm{S}_{\mathrm{pO}_{2}}$ and $\mathrm{P}_{\mathrm{ETCO}}$ were continuously monitored, while blood pressure was intermittently monitored. Propofol was administered at an initial rate $250 \mu \mathrm{g} / \mathrm{kg} / \mathrm{min}$ after time out procedure and then was adjusted downward until a stable plane of total intravenous anesthesia for endoscopy occurred, usually $50-75 \mu \mathrm{g} / \mathrm{kg} / \mathrm{min}$ by case mid-point. Remifentanil 10-30 $\mu \mathrm{g}$ aliquots were used as an adjunct to provide analgesia in a subset of subjects. Remifentanil was used at the discretion of the anesthesiologist and primarily at the beginning of the procedure to facilitate endoscope insertion or to acquire multiple biopsies if propofol alone was not sufficient. Specifically, total intravenous anesthesia with propofol was adjusted on the high end (increased) if the subjects were still responsive to questions or intolerant to insertion of bite block. Total intravenous anesthesia with propofol was adjusted on the low end (decreased) in a variety of scenarios: if bradypnea occurred with the breathing frequency decreasing by $50 \%$ or $<4$ breaths/min; if any period of apnea lasted $>30 \mathrm{~s}$, in which case infusion was stopped temporarily; if oxygen saturation decreased to $<93 \%$ with $>4 \mathrm{~L} / \mathrm{min}_{2}$ by face mask or nasal canula; if hypotension occurred with sys- 
tolic blood pressure $<90 \mathrm{~mm} \mathrm{Hg}$ or $<70 \%$ baseline; or if bradycardia occurred with heart rate $<60$ beats/min. If the subject was nonresponsive or apneic, jaw thrust maneuvers were performed. In these instances, oxygen flow would be increased to $10 \mathrm{~L} / \mathrm{min}$ by face mask. If $\mathrm{S}_{\mathrm{pO}_{2}}$ decreased to $<85 \%$ for $>5 \mathrm{~s}$, ventilation via bag-valve mask technique using $100 \%$ oxygen would be used. Total intravenous anesthesia in the intervention group followed the same protocol, but anesthesiologists also monitored the RVM and tried to maintain $\dot{\mathrm{V}}_{\mathrm{E}} \%$ in the $40-80 \%$ range as indicated by the yellow range on the RVM screen. The only rule added to the protocol was to decrease total intravenous anesthesia titration before $\dot{\mathrm{V}}_{\mathrm{E}} \%$ decreased to $<40 \%$ and to increase titration if $\dot{\mathrm{V}}_{\mathrm{E}} \%$ increased to $>80 \%$. No subjects received intravenous hemodynamic support for this study.

\section{Detecting Changes in Hypoventilation and Apneas}

$\dot{\mathrm{V}}_{\mathrm{E}} \%$ was measured throughout the procedure and during emergence from anesthesia. To compare $\dot{\mathrm{V}}_{\mathrm{E}} \%$ from procedures of different durations, each procedure was divided into 4 equal time periods, and the average $\dot{\mathrm{V}}_{\mathrm{E}} \%$ was calculated for each quartile. The first quartile represented the period of induction where we expected to see the least amount of respiratory depression, the second and third quartiles represented the period of deepest sedation, and the fourth quartile represented the recovery period at the end of the procedure. Hypoventilation was defined as $\dot{\mathrm{V}}_{\mathrm{E}} \%<40 \%$ at any time throughout the procedure. The amount of time with $\dot{\mathrm{V}}_{\mathrm{E}} \%<40 \%$ was added up and normalized by the length of each procedure to obtain the percentage of time with hypoventilation for each subject. The $40 \%$ threshold has previously been used as a conservative cutoff for unsafe ventilation when compared to the predicted $\dot{V}_{E}$ of the subject based on a formula with body surface area and sex. ${ }^{16,17}$ In this case, $\dot{\mathrm{V}}_{\mathrm{E} \text {-baseline }}$ was used instead of the predicted $\dot{V}_{\mathrm{E}}$ because it represented a characteristic measure of normal breathing for each subject without additional calibration with a spirometer. Apneic episodes were defined as a period of at least $15 \mathrm{~s}$ during which $\dot{\mathrm{V}}_{\mathrm{E}} \%$ was equal to zero, corresponding to no breath detected by the RVM.

\section{Statistical Analysis}

Demographics and clinical characteristics data were compared using either Fisher exact test (when comparing categorical variables) or 2-sample $t$ tests (when comparing continuous variables). Unpaired 2-sample $t$ tests were also used to calculate the effect of the RVM intervention on measured $\dot{V}_{\mathrm{E}} \%$ across the 2 groups. To compare the effect of the control and intervention groups on $\dot{\mathrm{V}}_{\mathrm{E}} \%$ over time, a 2-way analysis of variance (ANOVA) was used with a
Table 1. Subject Demographics and Clinical Characteristics

\begin{tabular}{|c|c|c|c|}
\hline Characteristics & $\begin{array}{l}\text { Control } \\
(n=38)\end{array}$ & $\begin{array}{c}\text { RVM } \\
\text { Intervention } \\
(n=27)\end{array}$ & $P$ \\
\hline \multicolumn{4}{|l|}{ Gender, $n(\%)$} \\
\hline Male & $22(58)$ & $15(56)$ & $.99 *$ \\
\hline Female & $16(42)$ & $12(44)$ & \\
\hline \multicolumn{4}{|l|}{ Type of procedure, $n(\%)$} \\
\hline Upper gastrointestinal endoscopy & $16(42)$ & $6(21)$ & $.24 *$ \\
\hline Lower gastrointestinal endoscopy & $16(42)$ & $15(58)$ & \\
\hline Both & $6(16)$ & $6(21)$ & \\
\hline \multicolumn{4}{|l|}{ ASA class, $n(\%)$} \\
\hline I & $1(3)$ & $2(7)$ & $.63^{*}$ \\
\hline II & $23(60)$ & $14(52)$ & \\
\hline III/IV & $14(37)$ & $11(41)$ & \\
\hline Age, y & $59 \pm 18$ & $62 \pm 10$ & $.45 \dagger$ \\
\hline Body mass index, $\mathrm{kg} / \mathrm{m}^{2}$ & $27 \pm 22$ & $32 \pm 22$ & $.38 \dagger$ \\
\hline Procedure duration, min & $29 \pm 18$ & $31 \pm 21$ & $.68 \dagger$ \\
\hline \multicolumn{4}{|l|}{ Anesthetics } \\
\hline Received remifentanil, $n(\%)$ & $25(66)$ & $19(70)$ & $.79 *$ \\
\hline Total propofol, mg & $270 \pm 172$ & $309 \pm 273$ & $.48 \dagger$ \\
\hline Total propofol, $\mu \mathrm{g} / \mathrm{kg} / \mathrm{min}$ & $75 \pm 48$ & $76 \pm 29$ & $.92 \dagger$ \\
\hline Total remifentanil, $\mu \mathrm{g}$ & $58 \pm 29$ & $51 \pm 15$ & $.25 \dagger$ \\
\hline Total remifentanil, $\mu \mathrm{g} / \mathrm{kg}$ & $0.72 \pm 0.39$ & $0.61 \pm 0.25$ & $.20 \dagger$ \\
\hline \multicolumn{4}{|c|}{$\begin{array}{l}\text { Data are presented as mean } \pm \text { SD unless otherwise specified. } \\
\text { * Fisher exact test. } \\
\dagger 2 \text {-sample } t \text { test. } \\
\text { RVM }=\text { respiratory volume monitor } \\
\text { ASA }=\text { American Society of Anesthesiologists }\end{array}$} \\
\hline
\end{tabular}

Bonferroni correction for the $P$ value. Normal distribution of data were confirmed using Lilliefors test prior to applying ANOVA or $t$ test. Frequency of apnea between the control and intervention groups was compared using the Wilcoxon rank-sum test, while the comparison of number of subjects with apnea between the 2 groups was performed with the Fisher exact test. Data are presented as mean $\pm \mathrm{SD}$, unless otherwise indicated, and $P<.05$ was considered significant.

In a preliminary cohort, the average $\dot{\mathrm{V}}_{\mathrm{E}} \%$ in the control group was found to be $65 \% \pm 26 \%$ (mean $\pm \mathrm{SD}, \mu_{1}=65 \%$, $\sigma_{1}=26 \%$ ). We anticipated that the use of the RVM in a group of similar subjects would increase average $\dot{\mathrm{V}}_{\mathrm{E}} \%$ by $20 \%\left(\mu_{2}=85 \%\right)$ with an equal SD of $26 \%\left(\sigma_{2}=26 \%\right)$. To demonstrate this increase with $80 \%$ power $\left(\mathrm{Z}_{\beta}=0.84\right)$ at a significance level of $P<.05\left(\mathrm{Z}_{\alpha / 2}=1.96\right)$, the required sample size for each group was calculated to be $27 .{ }^{18}$ We planned to recruit 70 subjects to have a sufficiently powered study, allowing for a $20 \%$ dropout rate.

\section{Results}

Sixty-five subjects (37 males) were enrolled in this randomized controlled trial (Table 1). All subjects underwent 
Table 2. $\quad \dot{\mathrm{V}}_{\mathrm{E}} \%$, Hypoventilation, and Apnea Outcomes

\begin{tabular}{|c|c|c|c|}
\hline Outcome Measurements & $\begin{array}{c}\text { Control } \\
(n=38)\end{array}$ & $\begin{array}{c}\text { RVM } \\
\text { Intervention } \\
(n=27)\end{array}$ & $P$ \\
\hline Average $\dot{\mathrm{V}}_{\mathrm{E}} \%$ & $64 \pm 37$ & $84 \pm 41$ & $.045^{*}$ \\
\hline $\begin{array}{l}\% \text { Time with hypoventilation } \\
\left(\dot{\mathrm{V}}_{\mathrm{E}} \%<40 \%\right)\end{array}$ & $32 \pm 24$ & $12 \pm 15$ & $<.001 *$ \\
\hline \multicolumn{4}{|l|}{ Apneas } \\
\hline $\begin{array}{l}\text { Total number of apneas all } \\
\text { subjects, } n\end{array}$ & 68 & 35 & \\
\hline $\begin{array}{l}\text { Median number of apneas } \\
\text { per subject, median (IQR) }\end{array}$ & $1(1-2)$ & $2(1-2)$ & $.037 \dagger$ \\
\hline Range of apneas, min-max & $0-4$ & $0-3$ & \\
\hline Subjects with apneas, $n(\%)$ & $35(92)$ & $21(78)$ & $.15 \ddagger$ \\
\hline \multicolumn{4}{|c|}{$\begin{array}{l}\text { Data are presented as mean } \pm \mathrm{SD} \text { unless otherwise specified. } \\
* 2 \text {-sample } t \text { test. } \\
\dagger \text { Wilcoxon rank-sum test. } \\
\ddagger \text { Fisher exact test. } \\
\mathrm{V}_{\mathrm{E}}=\text { expiratory minute volume } \\
\mathrm{RVM}=\text { respiratory volume monitor } \\
\mathrm{IQR}=\text { interquartile range }\end{array}$} \\
\hline
\end{tabular}

upper or lower gastrointestinal endoscopies under total intravenous anesthesia with propofol or, in some cases, remifentanil. Thirty-eight subjects were randomly placed into the control group (22 males, age $59 \pm 18 \mathrm{y}$, body mass index $27 \pm 22 \mathrm{~kg} / \mathrm{m}^{2}$ ), and the remaining 27 subjects were placed into the RVM intervention group (15 males, age $62 \pm 10 \mathrm{y}$, body mass index $32 \pm 22 \mathrm{~kg} / \mathrm{m}^{2}$ ). There was no statistically significant difference in the total amount of propofol delivered between the control group $(270 \pm 172 \mathrm{mg}, 75 \pm 48 \mu \mathrm{g} / \mathrm{kg} / \mathrm{min})$ and intervention group $(309 \pm 273 \mathrm{mg}, 76 \pm 29 \mu \mathrm{g} / \mathrm{kg} / \mathrm{min})$. Twenty-five subjects (66\%) in the control group and 19 subjects in the intervention group $(70 \%)$ received reminfentanil, but the total amounts were not different $(58 \pm 29 \mu \mathrm{g}$ vs $51 \pm 15 \mu \mathrm{g})$. No serious adverse events occurred in either group.

\section{Effects of Respiratory Monitoring on $\dot{\mathrm{V}}_{\mathrm{E}} \%$ and Hypoventilation}

Subjects in the intervention group had a significantly higher average $\dot{\mathrm{V}}_{\mathrm{E}} \%$ throughout the entire procedure compared to the control group ( $84 \pm 41 \%$ vs $64 \pm 37 \%$, $P=.045)$ (Table 2). Each procedure was divided into 4 equal time periods, and the average $\dot{\mathrm{V}}_{\mathrm{E}} \%$ was calculated for each quartile (Fig. 1). Across the entire subject cohort, we noted an expected decrease in $\dot{\mathrm{V}}_{\mathrm{E}} \%$ in response to total intravenous anesthesia. The average $\dot{\mathrm{V}}_{\mathrm{E}} \%$ for the control group decreased from $100 \%$ at baseline to as low as $53 \pm 24 \%$ in the third quartile before it recovered to $58 \pm 36 \%$ in the fourth quartile. In contrast, the average $\dot{\mathrm{V}}_{\mathrm{E}} \%$ in the intervention group decreased to only $74 \pm 41 \%$ in the third quartile before it recovered to $79 \pm 41 \%$ in the

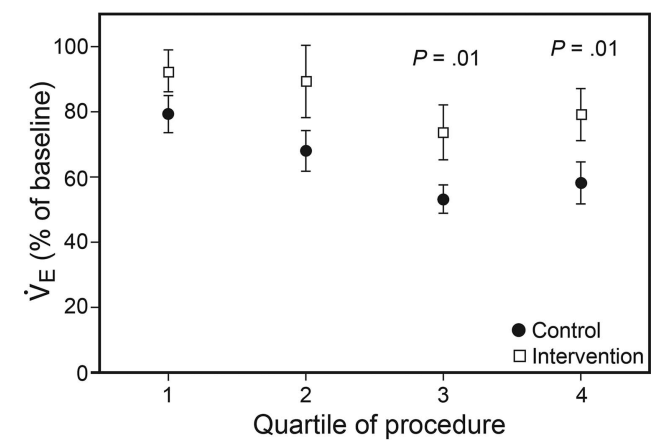

Fig. 1. Mean expiratory minute ventilation $\left(\dot{V}_{E}\right)$ and standard error of the mean are plotted for 4 equal periods of time for each procedure. Average $\dot{V}_{E}$ as a percentage of $\dot{V}_{E \text {-baseline decreased for }}$ both control $(n=38)$ and intervention $(n=27)$ groups during the first 3 quartiles of the procedure, corresponding to anesthesia induction and the majority of the endoscopic procedure. Average $\dot{V}_{E}$ decreased by $20 \%$ more in the control group compared to the intervention group for the third and fourth quartiles, respectively.

fourth quartile. The difference between the control and RVM intervention groups was statistically significant for both the third and fourth quartile $(P=.02$, 2-way ANOVA with Bonferroni correction). The decrease in $\dot{V}_{\mathrm{E}} \%$ throughout the procedure followed by an increase in the last quartile was consistent with total intravenous anesthesia titration and emergence from sedation.

Example trends of $\dot{\mathrm{V}}_{\mathrm{E}} \%$ and $\mathrm{f}$ for a subject from the control group (Fig. 2A) show a decrease in both $\dot{\mathrm{V}}_{\mathrm{E}} \%$ and $\mathrm{f}$ after administration of propofol. In this particular example, $\dot{\mathrm{V}}_{\mathrm{E}} \%$ dropped below the defined threshold for hypoventilation $\left(\dot{\mathrm{V}}_{\mathrm{E}} \%<40 \%\right)$ and remained below this threshold for $28 \%$ of the duration of the procedure. Conversely, the $\dot{\mathrm{V}}_{\mathrm{E}} \%$ of a subject in the RVM intervention group did not go below $40 \%$ throughout the entire procedure (Fig. 2B). Averaged across each group, control subjects experienced hypoventilation for $32 \pm 24 \%$ of the duration of each procedure, whereas subjects in the RVM intervention group experienced hypoventilation for only $12 \pm 15 \%$ of the time (Table 2). The use of the RVM to titrate total intravenous anesthesia resulted in a statistically significant $62 \%$ decrease of percentage of time subjects experienced hypoventilation $(P<.001,2$-sample $t$ test).

\section{Frequency of Apneic Episodes}

We noted episodes of apnea in both experimental groups; however, the median number of apneas per subject in the intervention group (median 1, interquartile range 1-2, maximum 3) was significantly lower than in the control group (median 2, interquartile range 1-2, maximum $4, P=.037$, Wilcoxon rank-sum test) (Table 2). The percentage of subjects experiencing apneas also decreased by $14 \%$ from the control group to the intervention group (92\% vs $78 \%$ of 

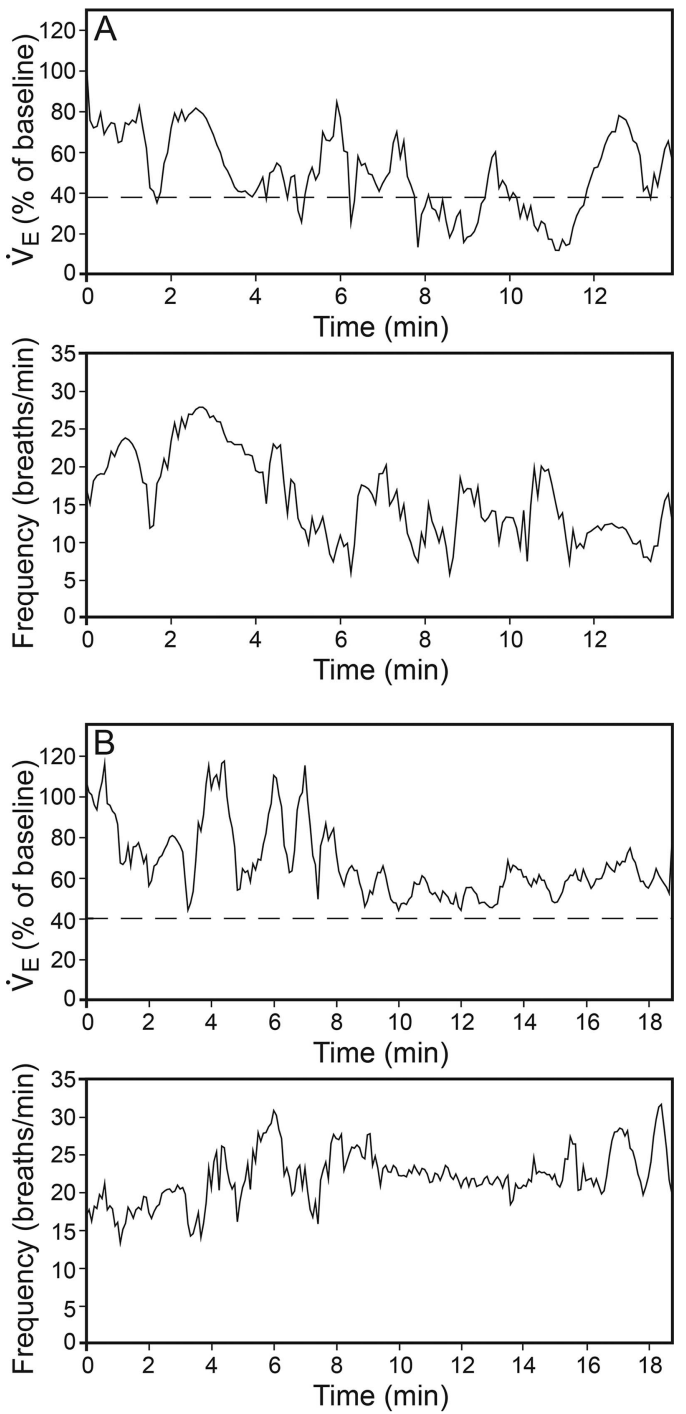

Fig. 2. Representative traces of $\dot{V}_{E}$ and breathing frequency for subjects in the control (A) and intervention groups (B). The control subjects spent more time below $40 \%$ of $\dot{\mathrm{V}}_{\mathrm{E} \text {-baseline }}$ (dashed horizontal lines) compared to the intervention example. Decreases in $\dot{V}_{E}$ do not necessarily correspond to decreases in breathing frequency.

subjects), although this effect was not statistically significant $(P=.15$, Fisher exact test).

\section{Discussion}

This study demonstrated the utility of respiratory monitoring with the RVM to direct anesthetic dosing in procedural sedation. Anesthesiologists in the intervention group were encouraged to titrate anesthetics to maintain $\dot{\mathrm{V}}_{\mathrm{E}}$ between $40 \%$ and $80 \%$ of $\dot{\mathrm{V}}_{\mathrm{E}-\text { baseline, }}$, a level of ventilation associated with both patient comfort and adequate ventilation. As expected, $\dot{\mathrm{V}}_{\mathrm{E}} \%$ decreased during the procedure in both the control and the RVM intervention groups; however, subjects in the RVM intervention group maintained a higher $\dot{\mathrm{V}}_{\mathrm{E}} \%$ on average, particularly in the second half of the procedure. Furthermore, subjects in the intervention group had a $62 \%$ decrease in the incidence of hypoventilation, defined as $\dot{\mathrm{V}}_{\mathrm{E}} \%<40 \%$. These results suggest that feedback from the RVM was clinically relevant to anesthesiologists and was successfully used to titrate anesthetic drugs to achieve appropriate levels of sedation while minimizing risk of respiratory depression. Notably, the total amount of propofol and reminfentanil was not significantly different between the control and the intervention groups and cannot alone explain the difference in $\dot{\mathrm{V}}_{\mathrm{E}} \%$ and hypoventilation between the groups. While the total amount of anesthetics may have been similar, the anesthesiologists using the RVM may have been able to detect hypoventilation earlier and intervene by adjusting the timing of the doses before $\dot{\mathrm{V}}_{\mathrm{E}} \%$ dropped to inadequate levels. Analysis of apneic episodes was performed post hoc and was not available at the time of the procedure. Nevertheless, the RMI intervention group had a $28 \%$ lower incidence of apneas per subject compared to the control group. Maintaining adequate levels of ventilation likely prevents oversedation, which can in turn lower the incidence of airway obstruction and apnea.

Recent randomized controlled trials suggest that the use of capnography in procedural sedation does not contribute to patient safety compared to standard care using pulse oximetry. ${ }^{11,12}$ Likewise, pulse oximetry has been shown to be a late indicator of respiratory depression. ${ }^{6}$ Both technologies are advocated by ASA guidelines for monitored anesthesia care despite their indirect measurement of ventilation. Closed-claims analysis by Berzin et $\mathrm{al}^{19}$ reveals that adverse respiratory events occurred in $14 \%$ of all examined cases undergoing procedural sedation despite the presence of capnography monitoring. In this study, both capnography and pulse oximetry were monitored during all procedures. The data presented here suggest that the impedance-based RVM could provide pertinent feedback of respiratory status to anesthesia providers and potentially mitigate the risk of respiratory depression. Furthermore, our data are consistent with Ebert et al, ${ }^{20}$ who observed significant hypoventilation in 18 of 20 subjects during gastrointestinal endoscopy, while increases in $\mathrm{P}_{\mathrm{ETCO}_{2}}$, albeit smaller in magnitude, occurred in only 10 of 20 subjects.

Several limitations of this study must be considered when interpreting these results. Randomization of the subjects was performed based on the day of the week of the scheduled procedure, which resulted in 11 more subjects being enrolled into the control group. Nevertheless, the required sample size was met for both the control and intervention groups. Outcomes such as hypoventilation were based only on RVM measurements and not compared to a secondary monitoring technique such as pulse 
oximetry or capnography. Previous studies, however, have compared the RVM to these technologies and established the use of the $40 \%$ threshold for determining safe ventilation. ${ }^{16,17}$ One of the main goals was to determine whether feedback from the RVM could be used to titrate total intravenous anesthesia to maintain $\dot{\mathrm{V}}_{\mathrm{E}} \%$; therefore, comparisons to other monitoring technologies was beyond the scope of this study. While we did not compare the depth of anesthesia between the 2 groups, there were no reports of awareness or procedure limitations from the gastrointestinal endoscopists suggesting inadequate levels of anesthesia. Likewise, the total amount of anesthetics dispensed was similar in the 2 groups. The anesthesiologists did not receive real-time alarms from the RVM in response to apnea, and the analysis of apneic episodes was done post hoc. While future versions of the RVM may alarm during apneic episodes, the current iteration only used $\dot{\mathrm{V}}_{\mathrm{E}} \%$ as an indicator of respiratory depression.

\section{Conclusions}

Until recently, direct measurement of ventilation has not been available in the endoscopic suite, constraining anesthesiologists and gastroenterologists alike to secondary indicators of adequate ventilation. Direct measurement of $\dot{\mathrm{V}}_{\mathrm{E}}$ by the RVM can potentially augment patient monitoring during procedural sedation and thus prevent respiratory complications.

\section{REFERENCES}

1. Inadomi JM, Gunnarsson CL, Rizzo JA, Fang H. Projected increased growth rate of anesthesia professional-delivered sedation for colonoscopy and EGD in the United States: 2009 to 2015. Gastrointest Endosc 2010;72(3):580-586.

2. Liu H, Waxman DA, Main R, Mattke S. Utilization of anesthesia services during outpatient endoscopies and colonoscopies and associated spending in 2003-2009. JAMA 2012;307(11):1178-1184.

3. Cohen LB, Benson AA. Issues in endoscopic sedation. Gastroenterol Hepatol 2009;5(8):565-570.

4. Bhananker SM, Posner KL, Cheney FW, Caplan RA, Lee LA, Domino KB. Injury and liability associated with monitored anesthesia care: a closed claims analysis. Anesthesiology 2006;104(2):228-234.

5. Metzner J, Posner KL, Domino KB. The risk and safety of anesthesia at remote locations: the US closed claims analysis. Curr Opin Anaesthesiol 2009;22(4):502-508.

6. Becker DE, Casabianca AB. Respiratory monitoring: physiological and technical considerations. Anesth Prog 2009;56(1):14-22.
7. MacLeod DB, Cortinez LI, Keifer JC, Cameron D, Wright DR, White WD, et al. The desaturation response time of finger pulse oximeters during mild hypothermia. Anaesthesia 2005;60(1):65-71.

8. Fu ES, Downs JB, Schweiger JW, Miguel RV, Smith RA. Supplemental oxygen impairs detection of hypoventilation by pulse oximetry. Chest 2004;126(5):1552-1558.

9. Beitz A, Riphaus A, Meining A, Kronshage T, Geist C, Wagenpfeil $\mathrm{S}$, et al. Capnographic monitoring reduces the incidence of arterial oxygen desaturation and hypoxemia during propofol sedation for colonoscopy: a randomized, controlled study (ColoCap Study). Am J Gastroenterol 2012;107(8):1205-1212.

10. Sivilotti ML, Messenger DW, van Vlymen J, Dungey PE, Murray HE. A comparative evaluation of capnometry versus pulse oximetry during procedural sedation and analgesia on room air. CJEM 2010; 12(5):397-404.

11. van Loon K, van Rheineck Leyssius AT, van Zaane B, Denteneer M, Kalkman CJ. Capnography during deep sedation with propofol by nonanesthesiologists: a randomized controlled trial. Anesth Analg 2014;119(1):49-55.

12. Campbell SG, Magee KD, Zed PJ, Froese P, Etsell G, LaPierre A, et al. End-tidal capnometry during emergency department procedural sedation and analgesia: a randomized, controlled study. World J Emerg Med 2016;7(1):13-18.

13. Voscopoulos C, Brayanov J, Ladd D, Lalli M, Panasyuk A, Freeman JE. Evaluation of a novel noninvasive respiration monitor providing continuous measurement of minute ventilation in ambulatory subjects in a variety of clinical scenarios. Anesth Analg 2013;117(1): 91-100.

14. Galvagno SM, Duke PG, Eversole DS, George EE. Evaluation of respiratory volume monitoring (RVM) to detect respiratory compromise in advance of pulse oximetry and help eliminate false desaturation alarms. J Trauma Acute Care Surg 2016;81(5 Suppl 2):S162S170.

15. Williams GW, George CA, Harvey BC, Freeman JE. A comparison of measurements of change in respiratory status in spontaneously breathing volunteers by the exspiron noninvasive respiratory volume monitor versus the capnostream capnometer. Anesth Analg 2017; 124(1):120-126.

16. Holley K, MacNabb CM, Georgiadis P, Minasyan H, Shukla A, Mathews D. Monitoring minute ventilation versus respiratory rate to measure the adequacy of ventilation in patients undergoing upper endoscopic procedures. J Clin Monit Comput 2016;30(1):33-39.

17. Voscopoulos C, Theos K, Tillmann Hein HA, George E. A risk stratification algorithm using non-invasive respiratory volume monitoring to improve safety when using post-operative opioids in the PACU. J Clin Monit Comput 2016;31(2):417-426.

18. Lachin JM. Introduction to sample size determination and power analysis for clinical trials. Control Clin Trials 1981;2(2):93-113.

19. Berzin TM, Sanaka S, Barnett SR, Sundar E, Sepe PS, Jakubowski $\mathrm{M}$, et al. A prospective assessment of sedation-related adverse events and patient and endoscopist satisfaction in ERCP with anesthesiologist-administered sedation. Gastrointest Endosc 2011;73(4):710717.

20. Ebert TJ, Middleton AH, Makhija N. Ventilation monitoring during moderate sedation in GI patients. J Clin Monit Comput 2017;31(1): 53-57. 